\title{
CLAUDE LEFORT Y EL CASO RUSHDIE (1995-1996) ${ }^{12}$
}

\author{
Beatriz Urías ${ }^{3}$
}

Résumé: Claude Lefort a présidé le Comité de défense de Rushdie en France de 1995 à 1996. Son intervention en faveur de Rushdie ne peut être considérée au seul registre de l'activisme mais a fait partie intégrante de son travail intellectuel. Tout d'abord parce que c'est plus d'une fois que les grands conflits internationaux du $\mathrm{XX}^{\circ}$ siècle ont nourri sa réflexion sur le Politique. Par ailleurs la fatwa lancée contre Rushdie le fut en raison de sa condition d'écrivain et l'on sait que les liens entre littérature et politique fut un des axes de la réflexion de Lefort. Cet essai souligne que les désaccords de Lefort avec Bernard-Henry Lévy et Salman Rushdie lui-même, n’obèrent en rien les reflexions sur ces liens que Lefort mena à partir de ces événements.

Mots-clès: Claude Lefort - Salman Rushdie - littérature - politique.

\section{Introducción}

A mediados de los años noventa, Claude Lefort aceptó presidir el Comité de defensa a Salman Rushdie en Francia. ${ }^{4}$ Este ensayo busca mostrar que su intervención en esta

1 Dossiê Claude Lefort: esse texto é parte do dossiê publicado a partir das comunicações realizadas no Colóquio Internacional Claude Lefort: a invenção democrática hoje, realizado na Universidade de São Paulo entre os dias 13 e 16 de outubro de 2015.

2 Este trabajo está basado en la consulta del Fondo Claude Lefort, localizado en el Centre d'Etudes Politiques Raymond Aron de l'Ecole des Hautes Etudes en Sciences Sociales. Este Fondo resguarda manuscritos de libros y artículos, que en su mayor parte son borradores de textos que aparecieron en libros; proyectos para cursos, seminarios y conferencias que Lefort impartió tanto en l'EHESS como en muy diversas universidades europeas, norteamericanas y latinoamericanas; correspondencia personal y profesional; informes de actividades en instituciones académicas; entrevistas publicadas en periódicos; sobretiros de artículos; manifiestos; notas de lectura sobre libros; invitaciones y propuestas para coloquios; algunas traducciones; finalmente, algunos expedientes sobre un mismo tema, entre ellos uno sobre el Comité Salman Rushdie. Además de la consulta de este Fondo, este ensayo retoma las ideas recogidas en una entrevista con Martine Poulain, una de las colaboradoras más activas dentro del Comité, en París durante el mes de diciembre de 2016. Agradezco a Gilles Bataillon los comentarios que hizo a esta versión.

3 Profesora Titular do Instituto de Investigaciones Sociales de la Universidad Nacional Autónoma de México. ${ }^{4}$ Martine Poulain advierte que el Comité de defensa a Salman Rushdie en Francia fue fundado en octubre de 1993: “Somme toute tardivement après la fatwa, à l'initiative d'un petit groupe de personnes (Anne Prassoloff et Régine Dhoquois, Pierre Pachet, enseignants à Paris 7, Driss El Yazami, animateur de l'Association Génériques, moi-même, rejoints notamment par l'écrivain Mashid Amir Shahi et le sociologue Ramine Kamrane, tous deux exiliés iraniens depuis la "révolution" Khomeiny), présidé par le philosophe Claude 
causa política internacional estuvo articulada con su obra intelectual y que los debates generados por el caso Rushdie son momentos importantes en la historia del pensamiento político de la segunda mitad del siglo XX. Parto de la consideración de que los grandes conflictos internacionales del siglo XX se convirtieron no pocas veces en el motor de su reflexión en torno a lo político. Entre ellos destacan la guerra de Argelia, la revolución húngara, el conflicto palestino-israelí, el Gulag y la causa de los disidentes soviéticos, la invasión de Checoeslovaquia, la situación política brasileña, el movimiento sindical en Polonia y la guerra de Kosovo.

En una carta que Alain Touraine dirigió a Claude Lefort en 2007 para agradecerle el envío del libro Le temps présent que acababa de ser publicado, Touraine expresa de manera emocionante y lúcida la forma en que los grandes acontecimientos de la historia política internacional del siglo XX se entretejen en el pensamiento de Lefort. Este libro es una compilación de muy diversos materiales - textos publicados, entrevistas, conferencias escritos por Lefort entre 1945 y $2005 .^{5}$

Me ha impactado descubrir en estas mil páginas sesenta años de historia que son, más o menos, aquellos que he vivido. ¿Cuántos pueden releer en un libro todos sus artículos y conferencias, alcanzando a identificar en ellos las pistas falsas y los errores de interpretación sin encontrar, sin embargo, errores de fondo? Lo que más me impresiona en esta recopilación es la manera en que se apega a los acontecimientos, aún a riesgo de perderse en ellos, demostrando muchas veces mejor que en un libro más formal, la fuerza de un trabajo de análisis que se mantiene inalterable. Como tú bien sabes, considero que eres el principal artífice de un redescubrimiento de lo político, que había sido relegado a un lugar secundario en la mayor parte de los análisis y no solamente en aquellos que llamábamos marxistas. Es cierto que Arendt fue una precursora, pero en Francia ha tenido que realizarse un trabajo gigantesco para avanzar en una reflexión en torno a lo político, y para lograrlo ha sido necesario que trabajaran, separados pero de manera convergente, espíritus tan diferentes como tú, Raymond Aron y François Furet. [.... Te has acercado al pensamiento falso, a la destrucción de la democracia, sin perder jamás la capacidad de análisis y también, lo que es esencial para

Lefort". Martine Poulain, "Vivre et écrire malgré la fatwa. Joseph Anton, une autobiographie, de Salman Rushdie". Bulletin des Bibliothèques de France, No.2, Paris, 2013. p. 3.

${ }^{5}$ Claude Lefort, Le temps présent. Écrits 1945-2005. Belin, Paris, 2007. 
mí, la capacidad y la voluntad de estar atento al surgimiento de movimientos libertarios, así como de identificar conflictos que estaban profundamente deformados por un pensamiento monolítico. Con toda mi amistad y mi admiración. ${ }^{6}$

Los grandes acontecimientos del siglo XX a los que se refiere Touraine representaron, en efecto, momentos a la vez concretos y privilegiados a partir de los cuales Lefort avanzó en la reflexión de cuestiones clave el relación al tema de lo político. Su interés por desentrañar el presente se vio reflejado en las polémicas públicas en las que intervino, así como en las líneas editoriales de las revistas que animó: Socialisme on barbarie (1948-1958); Texture (1971-1975); Libre (1977-1980); Passé-présent (1982-1984); y en menor medida Le temps de la réflexion (1980-1989). Su participación en el Comité de defensa a Salman Rushdie se sitúa en este contexto.

\section{El Comité Salman Rushdie}

El Comité de defensa a Rushdie en Francia (1995-1996) nació vinculado - gozando también de autonomía - frente al Comité internacional que se formó en Inglaterra bajo la cobertura de la organización Artículo 19, dedicada a la protección de periodistas y escritores perseguidos en todo el mundo. ${ }^{7}$ El punto de partida de las actividades del

\footnotetext{
6 "Quel choc se fut pour moi de découvrir ces mille pages qui défilent sur 60 ans d'histoire qui sont aussi, à peu de chose près, celles que j'ai vécu. Combien sont-ils ceux qui peuvent relire en plus de leur livre tous les articles et toutes les conférences qu'ils ont faites en y reconnaissant des fausses routes ou des erreurs d'interprétation mais sans jamais y trouver d'erreurs véritables. Car ce qui est le plus impressionnant dans ce livre est combien il se tient près des événements au risque de sembler s'y perdre mais en démontrant ainsi, souvent mieux que dans les livres plus construits, la puissance d'un travail d'analyse qui n'est jamais dementi. Comme tu le sais, je considère que tu es le principal artisan d'une redécouverte du politique qui avait été réleguée à une place sécondaire dans la plupart des analyses et pas seulement celles que l'on disait marxistes. Il est vrai que Arendt a éclairé le monde plus vite mais en France ce fut un gigantesque travail de faire comprendre le politique et pour y arriver il a fallu que travaillent séparément mais de manière convergente des esprits aussi différents que toi-même, Raymond Aron et François Furet. [....]Tu as reussi à t’approcher au plus près de la fausse pensée et de la destruction de la démocratie sans jamais perdre ta capacité d'analyse et aussi, et pour moi c'est l'essentiel, ta capacité et ta volonté de trouver et de montrer les mouvements de libération et les conflits qui étaient si profondement déformés par une pensée qui voulait avoir tous les monopoles à la fois. Avec toute mon amitié et mon admiration". Carta de Alain Touraine a Claude Lefort. Paris, 5 de febrero de 2007. Fondo Claude Lefort, CESPRA-EHESS.

${ }^{7}$ En el libro Joseph Anton. A Memoir, publicado en 2012, Rushdie relata en tercera persona lo que fue su identidad secreta bajo el seudónimo de Joseph Anton durante los años en los que estuvo amenazado por la fatwa. Describe los pormenores bajo los cuales su caso fue tomado por Artículo 19, en un momento en que la organización era presidida por Frances d'Souza y que el International Rushdie Defense Committee quedó a
} 
Comité en Francia fue la declaración que Rushdie había lanzado en Helsinki en 1992: "Por la primera vez, desde hace tres años y medio, tengo la impresión de haber tomado la iniciativa, de no cargar con la condena que se me ha impuesto. No pido compasión, sentimiento que terminaría por desgastarse, sino que se ejerza mayor presión". ${ }^{8} \mathrm{El}$ llamado a adherirse al Comité de apoyo al escritor en Francia, muy probablemente escrito por Lefort, retomaba esta idea:

Los miembros del Comité desean contribuir a ejercer esta presión. No les preocupa tanto la indiferencia hacia Rushdie como la aceptación de lo que ya aparece como una situación de hecho. Es por ello que han resuelto crear conciencia y mobilizar a un estrato sustancial de la opinión pública, así como presionar al gobierno para que intervenga ante las autoridades iranís a fin de que anulen la condena a Rushdie. ${ }^{9}$

El grupo encabezado por Lefort subrayó desde el inicio el carácter político - y no solamente cultural - de la fatwa decretada en contra de Rushdie. La estrategia básica de defensa se desprendió de los lineamientos promovidos a nivel internacional por la organización Artículo 19 en el sentido de defender no sólo la libertad de expresión del escritor, sino de articular un posicionamiento político europeo en relación a su caso. Algunos comunicados fechados en 1995 reiteran que la condena pronunciada por el Ayatollah Khomeiny en 1989 podría ser fácilmente revocada por el régimen iraní; y por otra parte, que la libertad de expresión y la defensa de la laicidad eran principios que muchos intelectuales musulmanes reclamaban y compartían. Señalaban también que lo que estaba en juego en el caso Rushdie era la defensa de la ley, por lo que sería muy

cargo de Carmel Bedford, quienes se convirtieron en sus aliadas políticas más cercanas: "The Committee had been set up quite independently of the person it was defending, to fight against "armed censorship", with the suppport of the Arts Council, PEN, the National Union of Journalists, the Society of Authors and the Writers Guild, and many other bodies. He (Salman Rushdie) had nothing to do with its origins; but as the years passed he (Rushdie) worked more and more closely with Frances and Carmel and they became his (Rushdie) indispensable political allies". Salman Rushdie, Joseph Anton. A Memoir. Vintage Books, London, 2013. p. 198.

8 "Pour la première fois, depuis trois ans et demi, j'ai le sentiment d'avoir pris l'initiative, de ne plus subir. Je ne demande plus la compassion, qui, à la longue, risque de se fatiguer, mais une pression plus forte". Citado en Claude Lefort, "Pourquoi un Comité de Défense de Salman Rushdie en France?". Fondo Claude Lefort, CESPRA-EHESS.

9 "Les membres du Comité souhaitent contribuer à exercer cette pression. Ils ne redoutent pas tant l'indifférence envers la personne de Rushdie que l'accoutumance à ce qui semble un état de fait. Ainsi sont-ils résolus à rechercher les moyens d'éclairer et mobiliser une large fraction de l'opinion publique et à presser le gouvernement d'intervenir auprès des autorités iraniennes pour qu'elles annulent la condamnation de Rushdie". Ibid. 
deseable que después del Senado americano y del Bundestag, el Parlamento francés adoptara una iniciativa a favor del escritor. Además de insistir en que Francia estaba obligada a declarar inaceptable el que un Estado terrorista - en este caso Irán - hiciera un llamado internacional para asesinar a un ciudadano británico, el Comité francés puso el acento en el peligro que entrañaba la debilidad o la ceguera de las democracias - europeas y no europeas - frente a una amenaza de tal magnitud en contra de sus principios e instituciones.

La importancia acordada al posicionamiento de la Unión Europea en relación al caso Rushdie explica que durante las visitas del escritor a Francia, el Comité optara por privilegiar los encuentros con personalidades políticas de alto nivel; entre ellas, Jacques Chirac, Edouard Balladur y Lionel Jospin. El objetivo era aprovechar el momento de la coabitación entre la derecha y la izquierda, así como jugar con las rivalidades generadas por la proximidad de las elecciones legislativas a fin de que los diferentes partidos políticos buscaran posicionarse frente a las organizaciones ciudadanas con la oferta más atractiva. El plan funcionó. Francia ocupaba en aquel momento la presidencia de la Unión Europea y gracias a la intervención del Comité de apoyo a Rushdie, se logró que Europa exigiera a Irán la suspensión de la fatwa. Esto es lo que en su momento se conoció como la "iniciativa francesa". Un agente clave para que esta iniciativa se concretara fue el director adjunto del gabinete del Ministro de Relaciones Exteriores Maurice Gordault-Montagne -, un indianista que admiraba la obra de Rushdie y con el cual el Comité entró en contacto. ${ }^{10}$

\section{Intervención política y reflexión filosófica}

El interés de Lefort por el caso Rushdie surgió desde el momento en que se dictó la fatwa en su contra en 1989, acerca de la cual muy pronto fijó su postura. En 1990 escribió un ensayo - "Humanisme et antihumanisme: homage à Salman Rushdie"11 - en el que desarrolló una serie de argumentos en contra del universalismo religioso, haciendo una defensa de los derechos de hombre. En 1993, Lefort publicaría un segundo artículo más corto en el periódico Libération - "Pour Rushdie, ecrivain"12 -, que era un llamado a que los intelectuales y la clase política francesa se comprometieran con la causa del escritor.

\footnotetext{
${ }^{10}$ Entrevista Martine Poulain-Beatriz Urías. París, diciembre 2016.

11 Claude Lefort, "Humanisme et antihumanisme: hommage a Salman Rushdie", Trabajo presentado en el coloquio "El hombre y la sociedad", Universidad de Lausana, mayo de 1990. Este trabajo fue después publicado en la Revue européenne des sciences sociales, tomo XXIX, No. 89. Droz, Genève, 1991; y más tarde quedó incluido en el libro Écrire. A l'épreuve du politique. Calmann-Lévy, Paris, 1992. pp. 37-54.

12 Claude Lefort, "Pour Rushdie, écrivain", Libération, 17 de junio de 1993. Posteriormente, este artículo fue incluido en el libro Le temps présent. Écrits 1945-2005, Op cit., pp. 711-714.
} 
Es interesante observar que la estrategia adoptada por el Comité de defensa a Salman Rushdie en Francia coincide plenamente con los argumentos que Lefort había desarrollado en los ensayos publicados en 1992 y 1993; y que estos argumentos estuvieron, a su vez, estrechamente ligados a algunos de los temas que Lefort exploraba dentro de su reflexión filosófica en torno a la democracia moderna: humanidad y derechos del hombre, libertad de expresión, ciudadanía y laicidad.

Acerca de los derechos humanos, Lefort sostuvo que la amenaza de muerte lanzada en contra de un individuo de nacionalidad británica y de origen musulmán por parte de un "gobierno terrorista" imbuido de fanatismo religioso era una violación grave, sobre todo si se consideraba que esta violación no se había producido en el contexto de una guerra o de un estado de emergencia. Afirmaba también que la amenaza que pesaba sobre Rushdie representaba una infracción a los principios del derecho internacional, en la medida en que el gobierno iraní estaba pasando por alto leyes vigentes en los países no-islámicos. Finalmente, argumentaba que al atentar en contra de la integridad personal y las libertades básicas de Rushdie, la "teocracia universal" encabezada por Khomeiny intentaba destruir los principios mismos de la democracia liberal, lo cual tenía un enorme peso simbólico.

En otro plano, criticaba la indiferencia de los intelectuales franceses ante la amenaza que el universalismo islámico había lanzado hacia Rushdie y hacia los principios básicos de las democracias occidentales. Es importante recordar que en 1991 una serie de individuos cercanos a Rushdie sufrieron atentados graves: el traductor japonés fue asesinado y se suscitaron ataques hacia el traductor italiano y el editor noruego, que sobrevivieron. Los servicios secretos británicos estaban encargados de la seguridad personal del escritor, en tanto que los traductores y los editores de su obra quedaban totalmente desprotegidos a pesar de que también habían sido amenazados. ${ }^{13}$ Ante estos hechos, consideraba Lefort, era alarmante que los intelectuales franceses no se hubieran comprometido con la misma fuerza que en Inglaterra, Dinamarca, Suecia, Noruega, Holanda y Alemania. Y se preguntaba, ¿cómo explicar que en Francia el caso Rushdie hubiera convocado a tan pocos? Descartando que el origen del problema fuera la falta de sensibilidad, atribuía esta indiferencia al hecho de que una parte sustancial de la

\footnotetext{
${ }^{13}$ En su autobiografía, Rushdie da cuenta del miedo que cundió en el mundo de los libros durante estos años a causa de la fatwa declarada en su contra: "The fear that spread through the publishing industry was real because the threat was real. Publishers and translators were threatened by the fatwa, too. And yet the world of the books, in which free people made free choices, had to be defended. He (Rushdie) thought often that the crisis was like an intense light shining down on everyone's choices and deeds, creating a world without shadows, a stark unequivocal place of right and wrong action, good and bad choices, yes and no, strenght and weakness. In that harsh glare some publishers looked heroic while others looked spineless". Salman Rushdie, Op cit., pp. 150-151.
} 
intelectualidad francesa había adoptado ideas convencionales acerca de la tolerancia y el derecho a la diferencia, lo cual era un obstáculo para reconocer en la condena a Rushdie una manifestación patente de intolerancia por parte de un régimen sustentado en el fanatismo religioso. Rechazaba los argumentos relativistas de acuerdo con los cuales los occidentales no eran capaces de aquilatar el grado de ofensa que el escritor había infringido al Islam.

En una entrevista realizada en 1992 a propósito de la aparición del libro Ecrire a lépreuve du politique, Lefort profundiza en el tema del relativismo, definiéndolo como una de las "enfermedades" de nuestro tiempo. El argumento que Lefort desarrolla en esta entrevista es que en el origen del relativismo que reinaba en el mundo moderno se encontraba la duda suscitada por la desaparición de antiguas certezas: la religión, la existencia de un orden natural o la idea de una historia teleológica. El relativismo, afirmaba, "desencadena la imbecilidad" en la medida en que impide condenar regímenes o pueblos sometidos a una opresión violenta - como es el caso de Irán, Arabia Saudita o China - bajo el pretexto de que hay que respetar su "identidad". ${ }^{14}$ En relación al caso Rushdie, el relativismo representaba además una afrenta al pueblo iraní y a los musulmanes que luchaban en contra del despotismo y el fanatismo; el proyecto integrista de regeneración de la humanidad reivindicado por un segmento radical del Islam no era, en efecto, compartido por todos los musulmanes.

Finalmente, Lefort argumentó a favor de Rushdie en tanto que creador de una obra literaria. Observaba que no era como ciudadano o como individuo que Rushdie había escrito los Versos satánicos (1988), sino como creador de una obra que rebasaba intereses y preferencias personales; una obra, en suma, que era patrimonio de sus contemporáneos y de la posteridad. Desde esta perspectiva, no era como ciudadano ni como individuo que Rushdie había sido condenado, sino como autor de una obra literaria que pertenecía a la humanidad. Si la condena de Rushdie estaba ligada a su condición de escritor, era en cuanto tal que había que salir en su defensa, subrayando el hecho de que una obra literaria estaba por encima del juicio de cualquier autoridad, fuera ésta política o religiosa. Lefort señalaba que el núcleo de la obra de Rushdie tocaba a los problemas más agudos del mundo moderno: abordaba la cuestión de la laicidad y de la ruptura con el universalismo religioso y desde ahí reformulaba la naturaleza del vínculo social, la idea de "humanidad" y el concepto de "hombre" en nuestro tiempo. Los Versos satánicos, afirmaba, no eran blasfemos pero sí representaban una reafirmación en contra del fanatismo y una salvaguarda de la verdad, lo cual explica que hubieran suscitado el encono del grupo político a la cabeza de una teocracia.

14 Claude Lefort, "Le relativisme déchaine l'imbecilité", Le Quotidien de Paris, Paris, 4 de febrero de 1992. Entrevista publicada posteriormente en Le temps présent, Op cit, pp. 683-687. 
La importancia de la literatura y el tema del vínculo entre ésta y la filosofía política le apasionó sobre todo a partir de la publicación de Ecrire a lépreuve du politique en 1992, libro en el cual reunió un conjunto de ensayos dedicados a escritores como Maquiavelo, Tocqueville, Sade, Orwell y Rushdie, que conjugaron una preocupación por la experiencia de lo político y una reflexión sobre su manera de escribir. ${ }^{15}$ Lefort declaraba que su intención al acercarse a estos autores había sido "restituir la mobilidad de su pensamiento, seguirlos en los caminos - diferentes y contradictorios - que emprendieron", y desde ahí tratar de comprender la situación a partir de la cual cada uno de ellos había llegado a formular determinadas preguntas, a fin de apropiárselas y desde ahí interrogar el presente. ${ }^{16}$

\section{Desencuentros intelectuales: pusilanimidad y narcisismo}

La vida del Comité Salman Rushdie en Francia fue muy corta, apenas dos años, y estuvo atravesada por una serie de tensiones dentro del grupo, así como del Comité con otros intelectuales franceses y con Rushdie mismo. Por lo que toca a los problemas dentro del grupo, Martine Poulain observa que el Comité de defensa a Rushdie en Francia "tuvo una vida a la vez apasionante y difícil debido a las tensiones que existían entre sus miembros, mismas que obedecían tanto a las divergencias en el análisis de la situación, como a las enemistades personales". ${ }^{17}$ Pierre Pachet, otro de los participantes en el Comité, confirma que desde el inicio el Comité fue solicitado por todo tipo de actores políticos y que esto generó problemas internos. ${ }^{18}$ Una parte del grupo se inclinaba por la opción heredada de la participación en las viejas organizaciones de izquierda en el sentido de convocar a las mayorías para impactar a la opinión pública, desdeñando la interlocución con los actores políticos de alto rango más allá de que éstos pudieran favorecer la causa de Rushdie a nivel internacional. Otros consideraban que más que llamar a las masas, había que ser pragmáticos, incidir sobre el Estado y sus instituciones, volverse más eficientes y construir buenos expedientes en torno a casos específicos. $\mathrm{Al}$ presidir el Comité de defensa a Rushdie, Lefort optó por una estructura flexible orientada hacia la búsqueda del apoyo de personalidades políticas para promover reuniones parlamentarias, sin descartar la participación de otros actores. Es decir, además de llevar a cabo un trabajo especializado, sistemático y preciso, el Comité Rushdie buscó articular

\footnotetext{
${ }^{15}$ Claude Lefort, Ecrire à l'éprewve du politique. Opcit.

${ }^{16}$ Claude Lefort, "Le relativisme déchaine l'imbecilité", Op cit, p. 683.

${ }^{17}$ Martine Poulain, Op cit, p. 3.

18 Pierre Pachet, "Les comités de défense, l'opinion et les médias". Raison et conviction de l'engagement (ed. Michel Wieviorka). Éditions Textuel, Paris, 1998. pp. 111-137.
} 
acciones políticas amplias a partir de algunas convicciones básicas que mantuvieron unido al grupo de organizadores durante varios meses.

Otra fuente de tensiones durante los meses en que Lefort presidió el Comité de apoyo a Rushdie fueron los desencuentros con figuras importantes del medio intelectual francés. El Fondo Claude Lefort contiene algunas cartas dirigidas a personalidades políticas como Michel Rocard, todas ellas fechadas al inicio de 1995, convocándolos a firmar un manifiesto a favor de que el Parlamento se pronunciara a favor de Rushdie. Algunos de ellos parecen haber atendido al llamado, en tanto que otros mostraron indiferencia hacia las iniciativas del grupo o fueron presa de pusilanimidad. Es el caso de Paul Ricoeur, a quien Lefort dirigió una carta en abril 1995 reprochándole su falta de compromiso ante el caso Rushdie; en ella se disculpa al mismo tiempo por la brusquedad con que lo había tratado en un encuentro reciente:

«Su silencio, en respuesta a una invitación [....] del Comité de Defensa a Salman Rushdie me sorprendió, es cierto, al igual que la noticia de su viaje a Irán. Hasta ahora no alcanzo a comprender por qué prefirió ignorar el llamado que le dirigimos (no se trataba de obtener una firma para un manifiesto más, como los que circulan cada semana), ni por qué su presencia en el Centro Georges Pompidou habría sido incompatible con el apoyo que Usted desea dar a la solidaridad que estamos brindando a los intelectuales independientes en un país en el que se encuentran en dificultades. [Durante nuestro último encuentro] este sentimiento de decepción me hizo emplear palabras desagradables que lamento [...] Sin embargo, el desacuerdo entre nosotros persiste. Sin buscar entablar una polémica, le doy mi punto de vista. ${ }^{19}$

Finalmente, el grupo encabezado por Lefort fue sensible al oportunismo y al narcisismo manifiestos en un personaje mediático como Bernard-Henry Levy, así como en Rushdie mismo. La historia remonta a 1993, cuando Salman Rushdie hizo dos viajes a Francia en los que entró en contacto con Jack Lang y Bernard-Henry Levy. En 1995, regresaría a París una vez más para participar en los encuentros organizados por sus editores, uno en el Centro Pompidou y otro en el Salon du Livre, así como para asistir a las entrevistas que el Comité había concertado con figuras políticas de alto nivel. Durante

\footnotetext{
19 Votre silence, en réponse à une invitation [....] du Comité de Défense de Salman Rushdie m'avait, il est vrai, choqué tout autant que la nouvelle de votre séjour en Iran. Encore maintenant, d'ailleurs, je ne comprends ni pourquoi vous avez préféré ignorer l'appel que nous vous adressions (il ne s'agissait pas d'obtenir une signature à quelque nouveau manifeste, comme il en circule toutes les semaines), ni pourquoi votre présence au Centre Georges Pompidou était incompatible avec le témoinage que vous souhaitez donner de notre solidarité à des intellectuels indépendants dans un pays où ils se trouvent en difficulté. Quoi qu'il en soit, ma déception m’a amené à employer des termes désagréables que je regrette [...] Cependant notre désaccord demeure. Sans la moindre intention polémique, je vous donne mon avis. Carta de Claude Lefort a Paul Ricoeur, París, 5 de abril 1995. Fondo Claude Lefort, CESPRA-EHESS.
} 
este último viaje, el escritor relegaría al Comité a un segundo plano, atribuyendo a Jack Lang y a Bernard-Henry Levy el arduo trabajo de organización mediante el cual el Comité había logrado el apoyo de la Unión Europea. En su libro autobiográfico, Rushdie no menciona siquiera al grupo de intelectuales encabezados por Lefort. ${ }^{20}$ Martine Poulain tiene duras y justas palabras para Salman Rushdie, Jack Lang y Bernard-Henry Levy, definiendo su actuación durante este episodio como una demostración de egocentrismo y una búsqueda vulgar de exposición mediática. Denuncia claramente el hecho de que en su afán protagónico, Bernard-Henry Levy hubiera estado a punto de frustrar la llegada de Rushdie a Francia al alertar a los medios de comunicación la noche anterior a su llegada y sembrando la confusión durante la aparición de Rushdie en el Salon du Livre. ${ }^{21}$

A partir de 1996, la organización Artículo 19 con base en Londres dio por terminado el trabajo de apoyo a Rushdie. Esta decisión coincidió con el momento en que la fuerza y el entusiasmo que animaron inicialmente al Comité en Francia habían decaído y que la relación entre Rushdie y el grupo de intelectuales encabezados por Lefort se había fracturado. En marzo de 1996, Lefort envió una carta personal a Salman Rushdie en la cual le transmite su decepción ante la actitud que había adoptado en relación al Comité francés. Explicita también que ni Martine Poulain ni Pierre Pachet, ni él mismo eran militantes profesionales o especialistas en obtener entrevistas con personajes políticos, a pesar de que durante el último año habían tenido que consagrar su tiempo a ello. Y finalmente, reitera por qué había creído importante encabezar la defensa de su causa, a pesar de que durante su visita a Francia hubiera ignorado los esfuerzos que el Comité había llevado a cabo:

Ni los amigos que menciono (Martine Poulain, Pierre Pachet), ni yo mismo, somos militantes, ni tampoco profesionales en la defensa de causas nobles. Estos (defensores) hacen falta, tengo simpatía por ellos (y) en diversas ocasiones he escrito a favor de una u otra de estas causas. Sin embargo, no estoy hecho para transmitir mensajes que recuerden sus obligaciones a los grandes personajes [...] Quiero persuadirme que estas ingratas iniciativas son necesarias. Es cierto que yo no las imaginaba cuando acepté presidir el Comité francés. Pensaba sólo en una cosa: tratar de sensibilizar a una parte de la opinión - aún restringida - acerca de los diferentes ángulos de la condena que pesaba sobre Usted y que tenía un carácter único. Es decir, había que denunciar la ofensiva de un gobierno terrorista en contra del autor de dos

\footnotetext{
20 Véase, Salman Rushdie, Op cit, pp. 446-447.
}

${ }^{21}$ Martine Poulain, $\mathrm{Op}$ cit. 
grandes libros, la violación del derecho internacional y la amenaza lanzada sobre las democracias cuyos gobernantes eran incapaces de enfrentar; mostrar el peligro del integrismo, la gravedad de la intimidación hacia la población de origen musulmán en Francia; por último, mostrar que una novela, debido únicamente a las virtudes de la escritura literaria, podía tocar a todos aquellos que estaban concernidos por la libertad del individuo en el mundo moderno. Mi compromiso, creo, no ha sido en vano, ya que además de haberme confrontado a aquellos (los más peligrosos) que pregonan el relativismo cultural rehusándose a juzgar los "valores" de los iranís, a través de mis escritos y de la discusión en reuniones públicas tuve la oportunidad de oponerme a algunos intelectuales pretenciosos y de inteligencia muy limitada que argumentaban que la literatura no tenía nada que ver con la política (¿no llegué incluso a escuchar el argumento de que en Francia el arte estaba igualmente amenazado por la censura?); o bien otros que declaraban lo contrario, es decir que cada individuo perseguido en el mundo requería del mismo tipo de apoyo - lo que la celebridad de Rushdie tendía a hacer olvidar. [...] Actualmente, comienzo a temer que nuestro Comité se convierta en un pequeño engranaje dentro de la correa de transmisión que ha sido puesta en marcha entre las organizaciones gubernamentales e intergubernamentales. Pero mayor, y de otra naturaleza, es mi temor de que Usted lo considere de esta manera. Mi intención es comunicárselo. Me parece escandaloso que Usted no haya tenido la idea de consagrarnos aunque fuera una hora de su tiempo durante su última visita a París. Era natural su preocupación por aparecer sobre todo como el autor de una nueva novela. Entendible, aunque no agradable, fue la decisión impuesta en Beaubourg por las Ediciones Plon y la Fnac de no abrir un espacio a nuestro Comité entre los invitados. Pero que en estas condiciones Usted nos ignorara, no es correcto. Desde hace un año, no lo olvide, hemos desplegado esfuerzos considerables y vencido fuertes oposiciones, para organizar los encuentros con los principales responsables políticos y obtener que su conferencia de prensa tuviera lugar en el recinto de la Asamblea Nacional. Si este fuera el caso, me parecería un poco triste que quedáramos remitidos al estatuto de intendentes. ${ }^{22}$

22 «Ni les amis que j'évoque (Martine Poulain, Pierre Pachet), ni moi-même ne sommes des militants, ni 
El carácter "único" que Lefort confirió a la causa de Rushdie reenvía a algunos puntos básicos que atraviesan su reflexión en torno a lo político. La debilidad de las democracias occidentales ante el desafío de cualquier forma de autoritarismo - en este caso el integrismo - que imponga límites a libertades básicas, como eran adherirse a una creencia religiosa o elegir una cultura de origen. La violación del derecho internacional por parte de un gobierno terrorista que amenazaba la vida de un gran escritor, por el hecho de serlo. El peligro del relativismo cultural en tanto que un obstáculo para situarse ante la violación de los derechos humanos. La revaloración de la escritura literaria como un elemento capaz de tocar a todos aquellos que estaban concernidos por la libertad del individuo en el mundo moderno. Martine Poulain observa que la historia de los siguientes veinte años mostraría la justeza de estas convicciones.

d'ailleurs, des professionnels de la défense des nobles causes. Il en faut, j’ai pour eux de la sympathie. À plusieurs reprises, j’ai écrit pour soutenir telle ou telle de ces causes. Mais je ne suis pas fait pour porter des messages aux grands de ce monde, qui les rappellent à leur devoir. [....] Je veux me persuader encore que ces ingrates démarches sont nécessaires. C'est vrai, je ne les imaginais pas quand j'ai accepté de présider le Comité français. Je ne pensais qu'à une chose: tenter de rendre sensible une partie de l'opinion - fut-elle fort restreinte - aux différents aspects de la condamnation qui vous frappait et lui donnait un caractère unique. C'est-à-dire: dénoncer l'offensive menée par un gouvernement terroriste contre l'auteur de deux grands livres, la violation du droit international et le défi lancé aux démocraties que leurs gouvernements étaient incapables de relever; faire mesurer le péril de l'intégrisme, l'ampleur de l'intimidation à l'égard des populations d'origine musulmane en France; enfin, montrer comment un roman, par les seules vertus de l'écriture littéraire, pouvait toucher aux du doute et de la liberté de l'individu dans le monde moderne. Mon engagement, je crois, n'a pas été vain, car, tandis que je me suis heurté à ceux (les plus dangereux) qui pataugent dans la vase du relativisme culturel et se refusent à juger des "valeurs" propres aux Iraniens, j'ai eu l'occasion, par l'écrit et par la parole dans les réunions publiques de m'opposer à quelques intellectuels prétentieux et d'intelligence bornée qui clamaient que la littérature n'avait rien à voir avec la politique (n’ai-je pas entendu aussi que l'art était menacé en France par la censure?) ou bien à d'autres qui déclaraient, à l'inverse, que chaque individu persécuté dans le monde requérait une même solidarité - ce que la notoriété de Rushdie tendait à faire oublier. A présent, je commence a craindre que notre Comité ne devienne un petit rouage dans le systeme de courroie de transmission engrené sur les organisations gouvernamentales et intergouvernamentales. Mais plus grande, et d'un autre caractère, est ma crainte que vous ne nous considériez comme tel. Je vous en fais franchement part. Il m'a semblé chocant que vous n'ayiez pas eu l'idée de nous consacrer, ne fut ce qu'une heure de votre temps, lors de votre dernier séjour à Paris. Naturel etait votre souci d'apparaitre avant tout comme l'auteur d'un nouveau roman. Intelligible, sinon agreable, fut la decisión imposée à Beaubourg par des Editions Plon et la Fnac de ne pas faire place à notre Comité parmi les invitants. Mais, que vous même vous nous ignoriez, en la circonstance, n'est pas bien. Il y a un an, ne l'oubliez pas, nous avons déployé des efforts considérables, et du vaincre des réticenses tenaces jusqu'au dernier moment, pour vous faire rencontrer les principaux responsables politiques et obtenir que votre conférence de presse ait lieu dans l'enceinte de l'Assemblée Nationale. Je trouverais un peu triste, si c'était le cas, que vous nous assigniez au statut de l'intendance". Carta de Claude Lefort a Salman Rushdie, Paris, 5 de marzo de 1996. Fondo Claude Lefort, CESPRA-EHESS. 


\section{Comentario final}

Reflexionando en forma retrospectiva, Pierre Pachet escribió que, más allá de los logros o de los fracasos manifiestos en el corto plazo, experiencias como la formación del Comité Rushdie despertaron en algunos de los participantes en éste un auténtico deseo por aprender y descubrir la vida pública en tanto que un espacio de deliberación cuyos secretos se revelaban al intervenir sobre ellos. ${ }^{23}$ Desde esta perspectiva, no importa si la ceguera de Rushdie obedeció a una sobre-exposición en los medios de comunicación o al hecho de que al convertirse en una figura pública desarrolló una forma de narcisismo. Lo que interesa resaltar en relación a la participación de Lefort en el Comité de defensa al escritor es que ni el egocentrismo de Bernard-Henry Levy ni el de Rushdie mismo, invalidaron la reflexión sobre temas cruciales que elaboró a partir de esta experiencia. Su intervención en el caso Rushdie puede, en efecto, entenderse como un momento privilegiado que le permitió ampliar los límites de su pensamiento en torno a lo político.

Recuperar la perspectiva de Lefort en el sentido de entrelazar la intervención política a una reflexión sobre lo político y las desviaciones de la democracia es una tarea urgente en países como México, en donde la multiplicación de fenómenos de violencia, corrupción y crueldad requiere de una nueva mirada crítica sobre el presente que rebase su mero registro.

\section{Referências bibliográficas}

Fondo Claude Lefort, CESPRA-EHESS.

LEFORT, Claude. "Humanisme et antihumanisme: hommage a Salman Rushdie", In: Revue européenne des sciences sociales, tomo XXIX, n. 89. Droz, Genève, 1991.

"Pour Rushdie, écrivain”, Libération, 17 de junio de 1993. Le temps présent. Écrits 19452005. Belin, Paris, 2007, pp. 711-714.

. "Le relativisme déchaine l'imbecilité", In: Le Quotidien de Paris, Paris, 4 de febrero de 1992.

${ }^{23}$ Pierre Pachet, Op cit, p.137. 
LEFORT, C. Ecrire à léprewve du politique. Paris, Calmann-Lévy, 1992.

PACHET, Pierre. "Les comités de défense, l'opinion et les médias". Raison et conviction de l'engagement (ed. Michel Wieviorka). Éditions Textuel, Paris, 1998. pp. 111-137.

RUSHDIE, Salman. Joseph Anton. A Memoir. Vintage Books, London, 2013. 